\title{
HJET polarimeter in RHIC Run 2017
}

\author{
A.A. Poblaguev*; E. Aschenauer, G. Atoian, K.O. Eyser, H. Huang, Y. Makdisi, \\ W. Schmidke, and A. Zelenski \\ Brookhaven National Laboratory, Upton, NY 11973, USA \\ E-mail: poblaguevebnl . gov
}

\section{Alekseev and D. Svirida}

Institute for Theoretical and Experimental Physics, Moscow 117218, Russia

The Polarized Atomic Hydrogen Jet Target (HJET) Polarimeter commissioned in 2004 was designed to measure absolute polarization of proton beams in Relativistic Heavy Ion Collider (RHIC). HJET performance in RHIC Run 2017 ( $255 \mathrm{GeV}$ proton beams) will be discussed. The long term (1-100 days) stability of the measured effective analyzing power $\left\langle A_{N}\right\rangle$ was found to be $\sqrt{\left\langle\left(\delta \sigma_{A_{N}}^{\text {sys }}\right)^{2}\right\rangle} / A_{N} \leq 0.1 \%$. The systematic error in measurement of the RHIC beam average polarization of $P \sim 56 \%$ was evaluated to be $\sigma_{P}^{\text {syst }} / P \lesssim 0.5 \%$. The statistical error in such a measurement is defined by the number of detected $p^{\uparrow} p^{\uparrow}$ elastic events and for an 8 hours RHIC store it was typically at $\sigma_{P}^{\text {stat }} \approx 2 \%$. The elastic events statistics of about $10^{9}$ per RHIC beam accumulated during Run 2017 allowed us to make a detailed study of the proton-proton single-spin $A_{N}(t)$ and double-spin $A_{N N}(t)$ analyzing powers in the momentum transfer range $0.001<-t<0.020(\mathrm{GeV} c)^{2}$. Contributions of the hadronic single $r_{5}$ and double $r_{2}$ spin-flip amplitudes were isolated. Analysis of the inelastic background $p_{\text {beam }}^{\uparrow}+p_{\text {target }}^{\uparrow} \rightarrow X+p_{\text {target }}$ allowed us to experimentally evaluate the beam and target spin correlated asymmetries in these processes for $M_{X}-m_{p} \lesssim 400 \mathrm{MeV}$ and $t \sim-0.005(\mathrm{GeV} c)^{2}$. The data acquired during 3 weeks of Gold-Gold beams in RHIC allowed us to make a high statistics measurement of the $p^{\uparrow} \mathrm{Au}$ analyzing power at $E_{\mathrm{Lab}}=27.2 \mathrm{GeV}$.

XVII International Workshop on Polarized Sources, Targets \& Polarimetry

16-20 October 2017

Kaist, South Korea

\footnotetext{
* Speaker.

${ }^{\dagger}$ Work was supported by the US Department of Energy under contract No. DE-SC0012704. Funding is also provided from the RIKEN BNL Research Center.
} 


\section{Introduction}

A precise measurement of the colliding beams polarization is an important component of the Relativistic Heavy Ion Collider (RHIC) Spin Program [1] at Brookhaven National Laboratory. The Polarized Atomic Hydrogen Gas Jet Target (HJET) [2] commissioned in 2004 was designed to measure absolute polarization of $24-250 \mathrm{GeV} / \mathrm{c}$ proton beams at RHIC with systematic errors better than $\Delta P / P \lesssim 0.05$. Main upgrade of the polarimeter was done in 2015. It included the installation of new silicon detectors, increasing detector acceptance, and a new DAQ based on $250 \mathrm{MHz} 12$ bits WFD [3]. This, together with the development of new methods in data analysis, allowed us to reduce the systematic uncertainties of the beam polarization measurements to a sub-percent level.

The main purpose of HJET is measurement of absolute, RHIC store average polarization of the beams. Actually HJET is a part of RHIC polarimetry complex which provides essential beam polarization information to RHIC experiments. However, discussion of the experimental determination of the beams polarization profile and decay time is beyond the scope of this paper.

The main goal of the paper is to review systematic uncertainties in RHIC beams polarization measurements in the Run 2017 ( $255 \mathrm{GeV}$ proton beams). As an introduction to the discussion, an overview of the spin correlated asymmetries in elastic $p^{\uparrow} p^{\uparrow}$ scattering will be given. Also, the HJET performance in RHIC Run 2017 including monitoring of the proton beams polarization and a study of the spin correlated asymmetries in $p^{\uparrow} p^{\uparrow}$ and $p^{\uparrow}$ Au scattering will be briefly reviewed.

\section{High energy beam polarization measurement at HJET}

\subsection{Spin correlated asymmetries for elastic $p p$ scattering}

A recoil proton azimuthal angle dependence of the cross section for the elastic scattering of the vertically polarized protons is given by

$$
2 \pi \frac{d^{2} \sigma}{d t d \varphi}=\frac{1}{2 \pi} \frac{d \sigma}{d t}\left[1+A_{N} \sin \varphi\left(P_{j}+P_{b}\right)+\left(A_{N N} \sin ^{2} \varphi+A_{S S} \cos ^{2} \varphi\right) P_{b} P_{j}\right]
$$

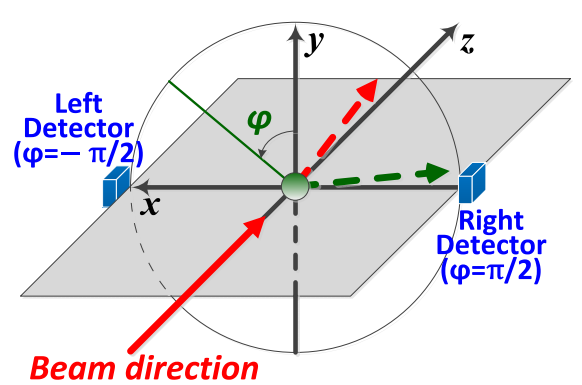

Figure 1: A schematic view of the $p^{\uparrow} p^{\uparrow}$ spin correlated asymmetries measurement. The recoil protons are measured in left/right symmetric detectors. Beam moves along $z$-axis. The transverse polarization axis is along the $y$-axis.

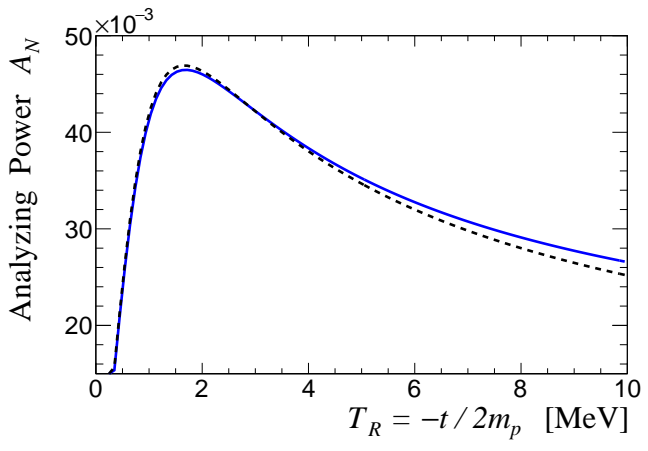

Figure 2: Single spin-flip analyzing power for elastic $p^{\uparrow} p$ scattering $\left(E_{\mathrm{Lab}}=255 \mathrm{GeV}\right)$. The dashed line is theoretical prediction $A_{N}^{\mathrm{QED}}(t)$ if no hadronic spin-flip amplitudes. The solid line corresponds to the preliminary HJET results in RHIC Run 2017. 
$P_{b}$ and $P_{j}$ are the beam and target (the Jet) polarizations, respectively. In Eq. 2.1, the sign of $P_{b}$ and/or $P_{j}$ is negative if the corresponding spin is directed down. The sign of the azimuthal angle $\varphi$ (see Fig. 1) is defined in accordance with Ann Arbor convention [4]. For the HJET configuration, $\sin \varphi_{R}=+1$ and $\sin \varphi_{L}=-1$ for the right and left detectors, respectively. Since $\cos \varphi=0$, the HJET measurements are insensitive to the $A_{S S}$. Single spin-flip, $A_{N} \sim 0.04$ (Fig. 2), and double spin-flip, $A_{N N} \sim 0.001$, analyzing powers are functions of the momentum transfer $t$ or, equivalently, the recoil proton kinetic energy $T_{R}$ since

$$
t=-2 m_{p} T_{R}
$$

where $m_{p}$ is proton mass.

The beam to Jet polarization ratio can be derived from the eight numbers of detected events in right/left (R/L) detectors depending on the beam $(\uparrow / \downarrow)$ and Jet $(+/-)$ spins up/down directions:

$N_{(R L)}^{(\uparrow \downarrow)(+-)}=N_{0}\left(1+\eta^{R L} \eta^{+-} a_{N}^{j}+\eta^{R L} \eta^{\uparrow \downarrow} a_{N}^{b}+\eta^{\uparrow \downarrow} \eta^{+-} a_{N N}\right)\left(1+\eta^{+-} \lambda_{j}\right)\left(1+\eta^{\uparrow \downarrow} \lambda_{b}\right)\left(1+\eta^{R L} \varepsilon\right)$

where spin correlated asymmetries are defined as

$$
a_{N}^{j}=\left\langle A_{N}\right\rangle P_{j}, \quad a_{N}^{b}=\left\langle A_{N}\right\rangle P_{b}, \quad a_{N N}=\left\langle A_{N N}\right\rangle P_{j} P_{b}
$$

$\lambda_{b, j}$ are the beam and Jet integrated intensity asymmetries, $\varepsilon$ is right/left acceptance asymmetry. Here, $\eta^{\uparrow \downarrow}$ means +1 for the beam spin up ( $\uparrow$ ) and -1 for the spin down $(\downarrow)$. The $\eta^{R L}$ and $\eta^{+-}$are defined similarly.

Equations 2.3 have an exact solution. For Jet spin correlated asymmetry,

$$
a_{N}^{j}=\frac{\sqrt{N_{R}^{\uparrow+} N_{L}^{\downarrow-}}+\sqrt{N_{R}^{\downarrow+} N_{L}^{\uparrow-}}-\sqrt{N_{R}^{\uparrow-} N_{L}^{\downarrow+}}-\sqrt{N_{R}^{\downarrow-} N_{L}^{\uparrow+}}}{\sqrt{N_{R}^{\uparrow+} N_{L}^{\downarrow-}}+\sqrt{N_{R}^{\downarrow+} N_{L}^{\uparrow-}}+\sqrt{N_{R}^{\uparrow-} N_{L}^{\downarrow+}}+\sqrt{N_{R}^{\downarrow-} N_{L}^{\uparrow+}}}
$$

and similar expressions for $a_{N}^{b}$ and $a_{N N}$. For other asymmetries,

$$
\lambda_{j}=\frac{\sqrt[4]{N_{R}^{\uparrow+} N_{R}^{\downarrow+} N_{L}^{\uparrow+} N_{L}^{\downarrow+}}-\sqrt[4]{N_{R}^{\uparrow-} N_{R}^{\downarrow-} N_{L}^{\uparrow-} N_{L}^{\downarrow-}}}{\sqrt[4]{N_{R}^{\uparrow+} N_{R}^{\downarrow+} N_{L}^{\uparrow+} N_{L}^{\downarrow+}}+\sqrt[4]{N_{R}^{\uparrow-} N_{R}^{\downarrow-} N_{L}^{\uparrow-} N_{L}^{\downarrow-}}}
$$

and similar expressions for $\lambda_{b}$ and $\varepsilon$.

If all asymmetries are small, the statistical errors for any pair of measured asymmetries are uncorrelated and are defined by the total statistics only:

$$
\sigma^{\text {stat }}=1 / \sqrt{N_{\text {tot }}}
$$

Since in HJET the same events are used to measure $a_{N}^{j}$ and $a_{N}^{b}$, the beam polarization can be related to known Jet polarization:

$$
P_{\text {beam }}=\frac{a_{N}^{\text {beam }}}{a_{N}^{\text {jet }}} P_{\text {jet }}
$$




\subsection{Analyzing power}

To determine beam polarization from Eq. 2.8 we, actually, do not need to know the analyzing power $A_{N}(t)$. This, obviously, is an advantage of the method because some systematic corrections are canceled in the ratio $a_{N}^{b} / a_{N}^{j}$. On other hand, a comparison of the measured $a_{N}^{j}(t) / P_{j}$ with expected $A_{N}(t)$ may serve as an indicator of a possible systematic error. Also, we can employ measured asymmetries $a_{N}^{\text {jet }}\left(T_{R}\right)$ and $a_{N}^{\text {beam }}\left(T_{R}\right)$ to adjust the theoretical description of the analyzing power $A_{N}(t)$.

Theoretically, single spin-flip analyzing power $A_{N}(t)$ is well studied [5]. The dominant contribution to $A_{N}(t)$ comes from interference of the electromagnetic spin-flip amplitude $\phi_{5}^{\mathrm{em}}(s, t)$ and the hadronic non-spin-flip amplitude $\phi_{+}^{\text {had }}(s, t)$

$$
A_{N}^{\mathrm{QED}}(t)=\frac{\sqrt{-t}}{m_{p}} \frac{\varkappa\left(1-\rho \delta_{C}\right) \frac{t_{c}}{t}}{\left(\frac{t_{c}}{t}\right)^{2}-2\left(\rho+\delta_{C}\right) \frac{t_{c}}{t}+1+\rho^{2}}
$$

where $\varkappa=\mu_{p}-1=1.793$ is proton's anomalous magnetic moment, $t_{c}=-8 \pi \alpha / \sigma_{\mathrm{tot}}$, and

$$
\delta_{C}=\alpha \ln \frac{2}{t\left(B+8 / \Lambda^{2}\right)}-\alpha \gamma
$$

$\left(\Lambda^{2}=0.71 \mathrm{GeV}^{2}\right.$ and $\gamma=0.5772$ is Euler's constant) is the Coulomb phase. The parameters $\rho(s)=\operatorname{Re} \phi_{+}^{\text {had }}(s, 0) / \operatorname{Im} \phi_{+}^{\text {had }}(s, 0), \sigma_{\text {tot }}(s)$ (total cross-section) and $B(s)$ ("the slope") are known from unpolarized $p p$ scattering experiments. In data analysis, we used the $255 \mathrm{GeV}$ values [6]:

$$
\rho=-0.009, \quad \sigma_{\mathrm{tot}}=39.19 \mathrm{mb}, \quad B=12 \mathrm{GeV} .
$$

The hadronic single spin-flip amplitude only slightly modifies the analyzing power

$$
A_{N}(t) / A_{N}^{\mathrm{QED}}(t)=1-2 \frac{\operatorname{Im} r_{5}-\delta_{C} \operatorname{Re} r_{5}+\left(\operatorname{Re} r_{5}-\rho \operatorname{Im} r_{5}\right) \frac{t}{t_{c}}}{\varkappa\left(1-\rho \delta_{C}\right)}=\alpha_{5} \times\left(1+\beta_{5} \frac{t}{t_{c}}\right)
$$

where $r_{5}=m_{p} \phi_{5}^{\text {had }} / \sqrt{-t} \operatorname{Im} \phi_{+}^{\text {had }}$. For the HJET momentum transfer range $-t \lesssim 0.02 \mathrm{GeV}$ we can neglect a possible $t$-dependence of the $\alpha_{5} \approx 1-1.1 \times \operatorname{Im} r_{5}$ and $\beta_{5} \approx 1.1 \times \operatorname{Re} r_{5}$.

For $255 \mathrm{GeV}$ beam energy, the hadronic spin-flip amplitude $r_{5}$ was experimentally isolated for the first time in RHIC Run 2017. The preliminary results are

$$
\operatorname{Re} r_{5}=\left(-7.1 \pm 0.5_{\text {stat }}\right) \times 10^{-3}, \quad \operatorname{Im} r_{5}=\left(20.2 \pm 2.4_{\text {stat }}\right) \times 10^{-3}
$$

The double spin-flip analyzing power can be parametrized as

$$
A_{N N}(t)=\frac{-2\left(\operatorname{Re} r_{2}+\delta_{C} \operatorname{Im} r_{2}\right) \frac{t_{c}}{t}+2 \operatorname{Im} r_{2}+2 \rho \operatorname{Re} r_{2}-\rho \frac{t_{c} \varkappa^{2}}{2 m_{p}^{2}}+\frac{2 t_{c} \varkappa}{m_{p}^{2}} \operatorname{Re} r_{5}}{\left(\frac{t_{c}}{t}\right)^{2}-2\left(\rho+\delta_{C}\right) \frac{t_{c}}{t}+1+\rho^{2}}
$$

where $r_{2}=\phi_{2}^{\text {had }} / \operatorname{Im} \phi_{+}^{\text {had }}$ and $\phi_{2}^{\text {had }}$ is hadronic double spin-flip amplitude. The preliminary results from RHIC Run 17 are

$$
\operatorname{Re} r_{2}=\left(-2.4 \pm 0.2_{\text {stat }}\right) \times 10^{-3}, \quad \operatorname{Im} r_{2}=\left(-0.4 \pm 0.1_{\text {stat }}\right) \times 10^{-3}
$$




\subsection{Overview of the leading order systematic uncertainties}

The ratio $a_{N}^{b} / a_{N}^{j}$ in Eq. 2.8 is actually a systematic error free measurement of the beam to Jet polarization ratio if Eqs. 2.3 are valid or, in other words, if the average analyzing power $\left\langle A_{N}\right\rangle$ is the same for left and right detectors, acceptance asymmetry $\varepsilon$ does not depend on the beam and target (Jet) spin direction, and $\delta P=\left|P^{\uparrow}\right|-\left|P^{\downarrow}\right|=0$ both for the beam and Jet.

However, even in this case the asymmetry $a_{N}$ is proportional to the effective analyzing power which, generally, may be affected by background and/or by uncertainties in energy calibration

$$
\delta A_{N}=\frac{b}{1+b}\left(\left\langle A_{N}^{(\mathrm{bgr})}\right\rangle-\left\langle A_{N}\right\rangle\right)-2 m_{p}\left\langle\frac{d A_{N}(t)}{d t} \delta T_{R}\right\rangle
$$

where $b$ is background to signal ratio and $A_{N}^{(\mathrm{bgr})}$ is effective analyzing power for background events. The values are averaged over the recoil energy range in the measurement. If $\delta A_{N}^{\text {jet }} \neq \delta A_{N}^{\text {beam }}$ the measured beam polarization is biased.

In a first order approximation, systematic errors in asymmetry measurements may be summarized as

$$
\begin{aligned}
& \delta a_{N}^{\text {syst }}=P \frac{\delta A_{N}^{(R)}+\delta A_{N}^{(L)}}{2}+\frac{\delta \varepsilon_{R}-\delta \varepsilon_{L}}{2} \\
& \delta \lambda^{\text {syst }}=P \frac{\delta A_{N}^{(R)}-\delta A_{N}^{(L)}}{2}+\frac{\delta \varepsilon_{R}+\delta \varepsilon_{L}}{2}
\end{aligned}
$$

where $\delta A_{N}^{(L, R)}$ are corrections to the effective analyzing power and $\delta \varepsilon_{L, R}$ are the spin correlated corrections to the acceptance asymmetries in the left/right detectors, respectively.

To suppress systematic errors in a spin asymmetry measurement, we need a good control for backgrounds, precise energy calibration, and the acceptance independence of the beam/Jet spins. In HJET, there is practically no correlation between acceptance and the beam polarization, $\delta \varepsilon_{L, R}^{\text {beam }}=0$. However, a correlation was observed between electronic noise in HJET detectors and RF transition frequency which managed Jet spin state. In turn, such a dependence can result in event selection efficiency (i.e. detector acceptance) on Jet spin.

\subsection{Instrumentation to indicate systematic errors}

In HJET, the beam polarization can be measured as a function of the recoil proton energy $T_{R}$, but the beam polarization must not depend on the $T_{R}$ :

$$
\frac{d P\left(T_{R}\right)}{d T_{R}}=\frac{d}{d T_{R}}\left(\frac{a_{N}^{\text {beam }}\left(T_{R}\right)}{a_{N}^{\text {jet }}\left(T_{R}\right)}\right)=0
$$

Obviously, an observation of the measured polarization dependence on $T_{R}$ will be a clear indication of systematic errors in the measurement.

Another important test for systematic errors is the $T_{R}$ dependence of measured integrated intensity asymmetries

$$
\frac{d \lambda_{\text {jet }}\left(T_{R}\right)}{d T_{R}}=\frac{d \lambda_{\text {beam }}\left(T_{R}\right)}{d T_{R}}=0
$$


If only one of four first order corrections $\delta A_{N}^{(L, R)}$ (2.17), $\delta \varepsilon_{L, R}$ (2.18) is non-zero then the measured $\lambda\left(T_{R}\right)$ allows one to calculate the $T_{R}$-dependence of the systematic error in the spin asymmetry measurement $a_{N}\left(T_{R}\right)$.

The normalized asymmetry

$$
\tilde{a}_{N}\left(T_{R}\right)=a_{N} / A_{N}^{\mathrm{QED}}=\left(P \alpha_{5}\right) \times\left(1-\beta_{5} \frac{T_{R}}{T_{c}}\right)
$$

$\left(T_{c}=-t_{c} / 2 m_{p}\right)$ has to be a linear function of recoil proton energy $T_{R}$. An important feature of the $\tilde{a}_{N}$ is that the slope $\beta_{5}$ has to be the same for the beam and Jet measured asymmetries.

Since there are 7 variables in 8 equations (2.3), there is a combination of measured number of events which has to be equal to 0 :

$$
b_{N N}\left(T_{R}\right)=\frac{\sqrt[4]{N_{R}^{\uparrow+} N_{R}^{\downarrow-} N_{L}^{\uparrow+} N_{L}^{\downarrow-}}-\sqrt[4]{N_{R}^{\uparrow-} N_{R}^{\downarrow+} N_{L}^{\uparrow-} N_{L}^{\downarrow+}}}{\sqrt[4]{N_{R}^{\uparrow+} N_{R}^{\downarrow-} N_{L}^{\uparrow+} N_{L}^{\downarrow-}}+\sqrt[4]{N_{R}^{\uparrow-} N_{R}^{\downarrow+} N_{L}^{\uparrow-} N_{L}^{\downarrow+}}}=0
$$

Obviously, a measurement of $b_{N N}\left(T_{R}\right)$ is a test for possible systematic errors.

\section{HJET polarimeter}

The Polarized Atomic Hydrogen Gas Jet Target (Fig. 3) consists of three main components: an atomic beam source, a Breit-Rabi polarimeter to measure hydrogen atoms polarization, and a recoil spectrometer to measure the beam and Jet spin correlated asymmetries in the recoil protons detection. Polarizations of both RHIC beams, blue and yellow are measured simultaneously.

The proton polarization in Jet Hydrogen atoms is defined by the strength $(1.2 \mathrm{kG})$ of the holding field magnet and is known with a high accuracy

$$
P_{\text {jet }}=0.957 \pm 0.001
$$

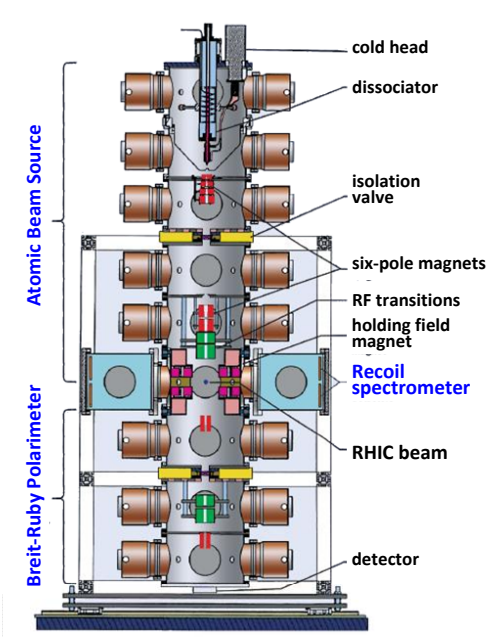

Figure 3: The Polarized Atomic Hydrogen Gas Jet Target at RHIC.

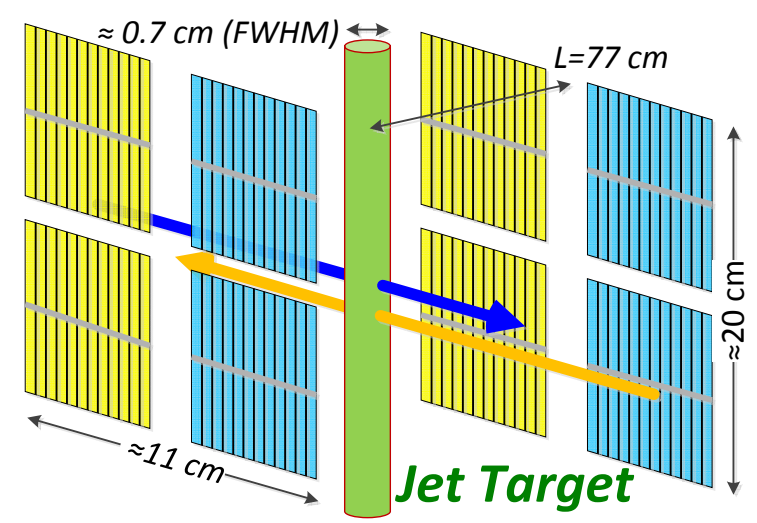

Figure 4: A schematic view of HJET polarimeter. 8 Silicon detectors, 12 readout channels each, are optionally referred as blue and yellow depending on which RHIC beam they measure. 
To minimize the effect of magnetic field on recoil protons position in the detactors, opposing Helmholtz coils were used. Jet density profile in the horizontal direction is well approximated by a Gaussian distribution $(\sigma \approx 2.6 \mathrm{~mm})$ with $1.2 \times 10^{12}$ atoms $/ \mathrm{cm}^{2}$ in the center. In RHIC Run 2017, Jet polarization was reversed every 10 minutes.

\subsection{Recoil spectrometer}

The recoil spectrometer is sketched in Fig. 4. To detect recoil protons, we use 8 pairs of Si wafers (12 vertically oriented strips of $3.75 \times 45 \mathrm{~mm}^{2}$ size, $470 \mu \mathrm{m}$ thickness, $\sim 0.37 \mathrm{mg} / \mathrm{cm}^{2}$ uniform dead-layer). For elastic scattering, the spectrometer geometry allows us to detect recoil protons with kinetic energy up to $T_{R} \approx 10-11 \mathrm{MeV}$ which corresponds to momentum transfer $-t=$ $2 m_{p} T_{R} \lesssim 0.020(\mathrm{GeV} / c)^{2}$. Protons with energy above $7.8 \mathrm{MeV}$ punch through the Si detector (only part of the proton kinetic energy is detected).

For signal readout we use 12 bit $250 \mathrm{MHz}$ FADC250 wave-form digitizers [7]. A full waveform (80 samples) is recorded for every signal above $\sim 0.5 \mathrm{MeV}$ threshold (Fig. 5). In the data analysis, the signal shape was parametrized by the following function

$$
W(t)=p+A\left(\frac{t-t_{i}}{n \tau_{s}}\right)^{n} \exp \left\{\left(-\frac{t-t_{i}}{\tau_{s}}+n\right)\right\}, t>t_{i}
$$

where $p$ is the pedestal, $A$ is signal amplitude, $t_{i}$ is signal start time, $n$ and $\tau_{s}$ are signal shape parameters. The waveform has maximum at time $t_{m}=t_{i}+n \tau_{s}$. At this time $W\left(t_{m}\right)=p+A$.

\subsection{Energy calibration of the Si detectors}

For energy calibration, all Si strips are exposed to $\alpha$-particles from two alpha-sources, ${ }^{148} \mathrm{Gd}$ (3.183 MeV) and ${ }^{241} \mathrm{Am}(5.486 \mathrm{MeV})$. A typical signal amplitude distribution in a Si strip is shown in Fig. 6.

Two different energies of $\alpha$-particles allow us to determine both the gain $g \sim 2.5 \mathrm{keV} / \mathrm{cnt}$ and dead-layer thickness $x_{\mathrm{DL}} \sim 0.37 \mathrm{mg} / \mathrm{cm}^{2}$ in every Si strip. Energy resolution $\sigma_{E} \sim 20 \mathrm{keV}$ is dominated by electronic noise.

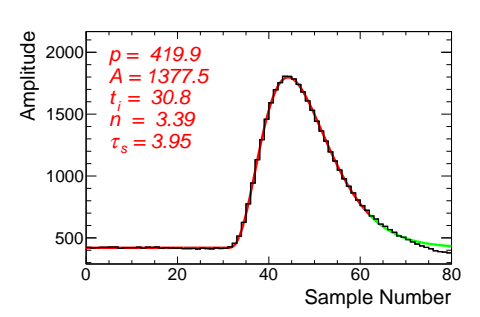

Figure 5: Signal waveform in HJET (black histogram). The red line indicates the time interval which is used in the waveform fit. The green line is the waveform function $W(t)$ beyond this interval. The sample time is about $4.1 \mathrm{~ns}$.

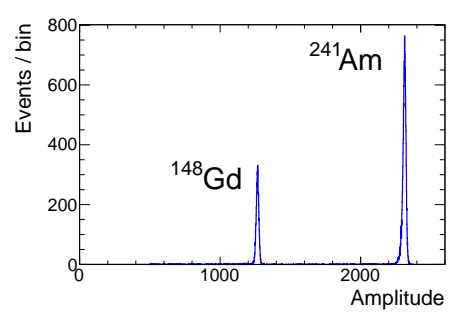

Figure 6: Signal amplitude distribution in the $\alpha$-source calibration. The ${ }^{241}$ Am line substructure (multiple peaks around $5.486 \mathrm{MeV}$ was accounted in the calibration.

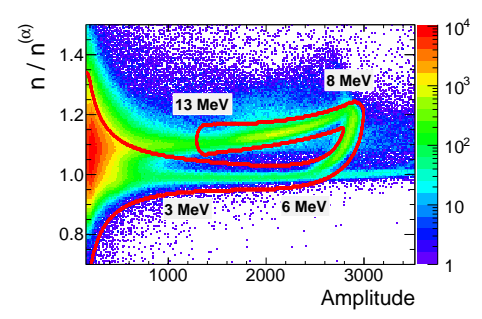

Figure 7: Event selection cut (solid red line) to separate punchthrough and stopped protons for the $0.5<T_{R}<13 \mathrm{MeV}$ energy range. No other cuts had been applied in this plot. 
To separate punch-through and stopped protons with the same measured signal amplitude, we analyzed the waveform shape. For that, the dependence of signal amplitude $A$ and waveform shape parameters $n$ and $\tau_{s}$ on proton kinetic energy $T_{R}$ was simulated $[3,8]$. The simulation parametrization was adjusted using $\alpha$-calibration data. For every pair of measured parameters $A$ and $n$ (within good event selection cut) the corresponding recoil proton kinetic energy was determined. The $A-n$ based event selection is illustrated in Fig. 7, $n^{(\alpha)}$ is the waveform shape parameter $n$ measured in $\alpha$-calibration.

\subsection{Event selection}

A typical measured time-amplitude distribution in a silicon strip is shown in Fig. 8. To study spin correlated asymmetries we have to isolate elastic events.

First, we have to verify that the detected particle is a proton. For that, we compare the measured signal time $t$ with expected time for recoil proton kinetic energy $T_{R}$ (derived from measured amplitude $A$ )

$$
\delta t=t-t_{0}-\text { tof }=t-t_{0}-\frac{L}{c} \sqrt{\frac{m_{p}}{2 T_{R}(A)}}
$$

$L=769 \mathrm{~mm}$ is the distance to detector, $c$ is speed of light, and $t_{0}$ is the time offset. Since the $\delta t$ distribution is dominated by the beam bunch longitudinal profile, it has to be the same for all $\mathrm{Si}$ strips.

Second, we have to verify that the missing mass $M_{X}$ (effective mass of the scattered beam proton) is equal to proton mass $m_{p}$. This condition may be written as

$$
z_{\text {strip }}-z_{\text {jet }}=L \sqrt{\frac{T_{R}}{2 m_{p}} \frac{E_{p}+m_{p}}{E_{p}-m_{p}-T_{R}}}=\kappa \sqrt{T_{R}}, \quad \kappa=17.9 \mathrm{~mm} / \mathrm{MeV}^{1 / 2}
$$

$z_{\text {strip }}$ and $z_{\text {jet }}$ are $z$-coordinates of recoil proton in the detector and in Jet (scattering point), respectively. For elastic scattering, the event rate dependence on recoil proton energy can be described as

$$
d N / d \sqrt{T_{R}} \propto \sqrt{T_{R}}(d \sigma / d t)_{e l} f(\kappa \delta \sqrt{T})
$$

where $f(z)$ is the jet target density profile and

$$
\delta \sqrt{T}=\sqrt{T_{R}}-\sqrt{T_{\text {strip }}}
$$
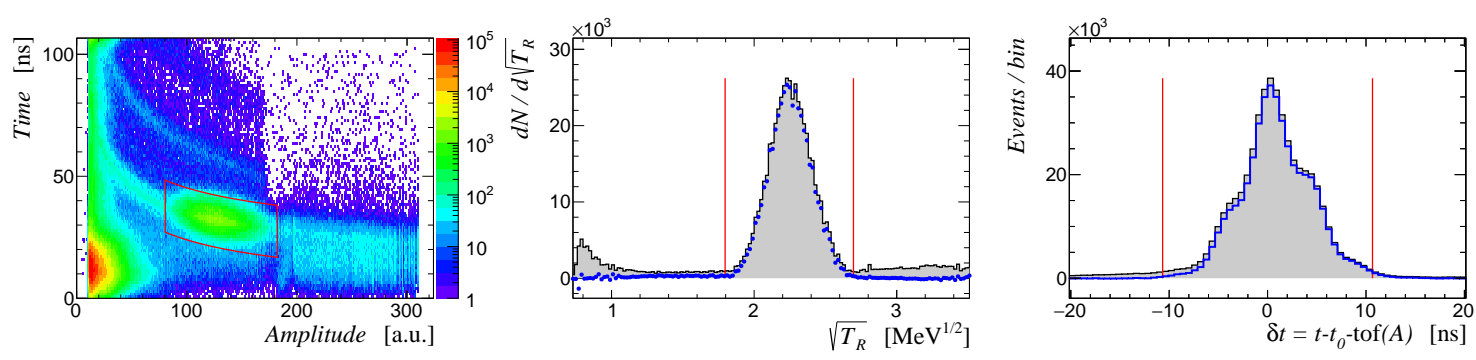

Figure 8: Elastic $p p$ events isolation. Event selection cuts are show by red lines. The $\delta \sqrt{T}$ cut is applied for events in the $\delta t$ histogram, and the $\delta t$ cut is applied in the $T_{R}$ histogram. 
$T_{\text {strip }}$ is recoil proton energy corresponding to the strip center $\left(\kappa \sqrt{T_{\text {strip }}}=\left\langle z_{\text {strip }}\right\rangle-\left\langle z_{\text {jet }}\right\rangle\right)$. Since the $\delta \sqrt{T}$ distribution is dominated by Jet density profile, it has to be the same for all Si strip. It is convenient to use the $\delta \sqrt{T}$ for the elastic event selection cut.

The efficiency of this cut may be affected by detector misalignment during installation and by corrections $\sim b_{\mathrm{MF}} / \sqrt{T_{R}},\left|b_{\mathrm{MF}}\right| \lesssim 1 \mathrm{~mm} \cdot \mathrm{MeV}^{1 / 2}$ due to recoil proton track bending in the holding magnetic field. A method to evaluate these corrections with accuracy $\sim 100 \mu \mathrm{m}$ was developed $[3,9]$ and was routinely used in the data analysis. This method can also be employed to monitor the time offset $t_{0}$ with accuracy $\sim 100 \mathrm{ps}$.

Two main sets of events selection cuts were used in RHIC Run 2017. The first one accounts as many elastic events as possible and, thus, minimize the statistical uncertainty of polarization measurement.

Cuts I: $\quad 0.6<T_{R}<10.6 \mathrm{MeV}, \quad|\delta t|<7 \mathrm{~ns}, \quad-0.40<\delta \sqrt{T}<0.40 \mathrm{MeV}^{1 / 2}$

The second set minimize the uncertainty in systematic corrections.

Cuts II: $\quad 2.0<T_{R}<9.5 \mathrm{MeV}, \quad|\delta t|<7 \mathrm{~ns}, \quad-0.18<\delta \sqrt{T}<0.30 \mathrm{MeV}^{1 / 2}$

Explanation of the cuts (3.8) will be given in section 4 .

\subsection{Background subtraction}

As one can see in Fig. 8 the $p p$ elastic signal contamination by background events is about several percent. We routinely applied a background suppression procedure to the analyzed data in order to eliminate background contribution to systematic errors. The method is based on the assumption that background $d N_{\mathrm{bgr}} / d T_{R}$ distribution is the same in all HJET Si strips. In this case (see Fig. 9), in every Si strip, events with recoil proton kinetic energy $T_{R}$ outside the elastic peak $|\delta \sqrt{T}|>0.6 \mathrm{MeV}$ could be used to determine (and subtract) the background under elastic peak in other Si strips.

For every detector, the background rate was determined as a function of $\delta t$ and $\sqrt{T_{R}}$. To properly account for possible spin correlated effects associated with background, every beam and Jet spin combination was evaluated separately. The background subtraction is demonstrated in Fig.
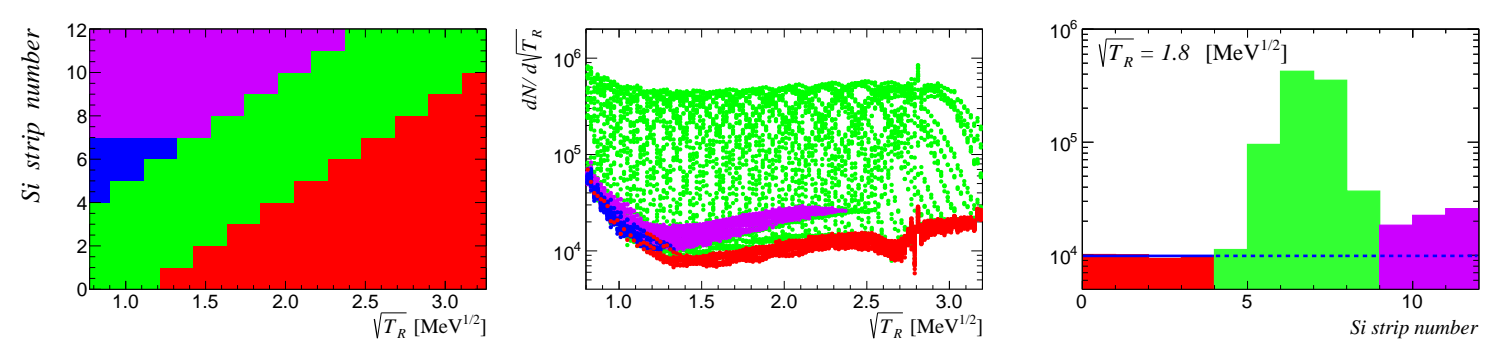

Figure 9: The superposition of $d N / s \sqrt{T_{R}}$ distributions for all Si strips (center). The markers colors depending on the strip location in a detector and recoil proton energy are explained in the left histogram. Green color is for elastic events, red and blue for backgrounds, and violet specifies the area contaminated by inelastic events. An example of background subtraction for fixed energy is shown in the right histogram. Background determined in the red area is extrapolated to the signal (green) area. 

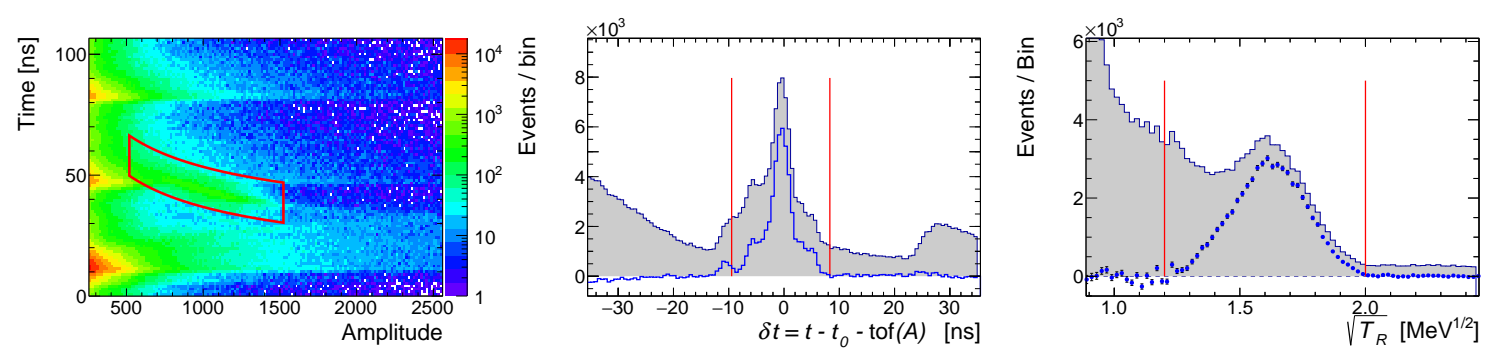

Figure 10: An example of the background subtraction in case of very high background. The gray filled histograms shows event distributions before background subtraction, the blue histograms are distributions after subtraction. The red lines show event selection cuts.

10. For illustration purposes we used non-usual data with very high background level from RHIC 2016 deuterium-Gold Run. Even in this extremal case the method works well. For the $255 \mathrm{GeV}$ $p p$-scattering, an example of background subtraction is shown in Fig. 8.

For the $255 \mathrm{GeV}$ proton beams, recoil protons from inelastic scattering $p+p \rightarrow X+p$ can be detected in HJET if $M_{X}-m_{p} \lesssim 400 \mathrm{MeV}$. Since such a background violate the assumption that $d N_{\text {bgr }} / d T_{R}$ is the same for all Si strips, the $z_{\text {str }}-\sqrt{T_{R}}$ area occupied by inelastic events (marked violet in Fig. 9) was excluded from the evaluation of the background level. Other possible sources of background subtraction inefficiency will be discussed below.

\section{Systematic uncertainties}

Since Jet polarization is very stable, a permanent measurement of the effective Jet spin asymmetry $\left\langle a_{N}^{\text {jet }}\right\rangle$ is a convenient way to monitor stability of HJET performance. The results of measurements with event selection Cuts I (3.7) of $\left\langle a_{N}^{\text {jet }}\right\rangle$ for every RHIC fill during Run 2017 are shown in Fig. 11. Unless a different will be explicitly specified, this paper will always use red and blue colors to display results obtained with yellow and blue beams, respectively.

One can see a perfect consistency between Jet spin asymmetry values measured with blue and yellow beams. Attributing the discrepancy between $\chi^{2}=263.4$ and NDF $=180$ to a possible fluctuation of the systematic error during the RHIC Run we have to conclude that the long term

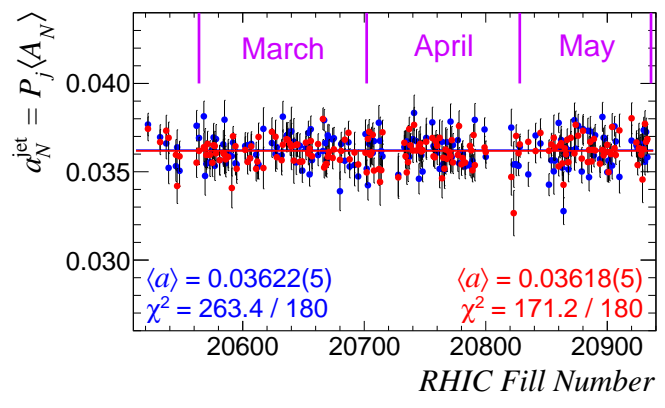

Figure 11: The measured RHIC store average Jet spin asymmetry during Run 2017.

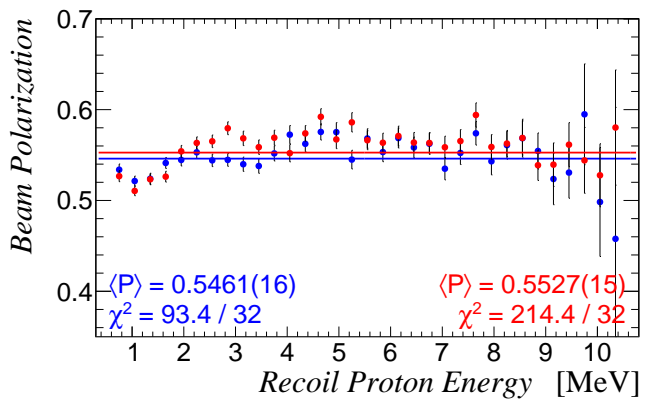

Figure 12: The measured beam polarization as a function or the recoil proton energy. 
stability of Jet asymmetry measurement does not exceed

$$
\left(\frac{\delta a_{N}}{a_{N}}\right)_{\text {long term }} \lesssim 0.1 \%
$$

This value includes possible fluctuations of Jet polarization, instability of the energy calibration, fluctuations of the signal contamination by backgrounds, etc. So, for a short term beam polarization measurement (Eq. 2.8) we can substitute the measured $a_{N}^{j}$ by the Run average $\left\langle a_{N}^{j}\right\rangle$ value:

$$
P_{\text {beam }}=\frac{a_{N}^{\text {beam }}}{\left\langle a_{N}^{\text {jet }}\right\rangle} P_{\text {jet }}\left(1+\delta_{\text {syst }}\right)=\frac{a_{N}^{\text {beam }}}{A_{N}^{\text {eff }}}
$$

$\delta_{\text {corr }}$ denotes a correction which has to be applied to compensate the systematic error. A necessity of such a correction for Cuts I is clearly followed from the observed dependence of measured beam polarization on the recoil proton energy (Fig. 12).

A very good stability of Jet spin asymmetry systematic error allows us to use the RHIC Run average effective analyzing power $A_{N}^{\text {eff }}$ and, thus, to minimize systematic uncertainties in polarization measurement but concurrently to keep statistical uncertainty as small as possible. For that we may employ some special set of event selection cuts (Cuts II, 3.8) for which the systematic correction $\tilde{\delta}_{\text {corr }}$ can be precisely determined and, then, to calculate

$$
A_{N}^{\text {eff }}=\frac{\left\langle\tilde{a}_{N}^{\text {jet }}\right\rangle}{P_{\text {jet }}\left(1+\tilde{\delta}_{\text {corr }}\right)} \frac{\left\langle a_{N}^{\text {beam }}\right\rangle}{\left\langle\tilde{a}_{N}^{\text {beam }}\right\rangle}
$$

To destinguish between Cuts I and Cuts II measurements, the latter were denoted by tilde.

\subsection{Noise dependence on Jet spin state}

It was found that HJET weak field transition (WFT) induces the $14 \mathrm{MHz}$ noise in some detectors. This noise (see Fig. 13) makes the efficiency of event selection cuts Jet state dependent (especially for low $T_{R}$ ) and, thus, may results in non-zero systematic corrections $\delta \varepsilon_{L, R}$ (Eqs. 2.17,

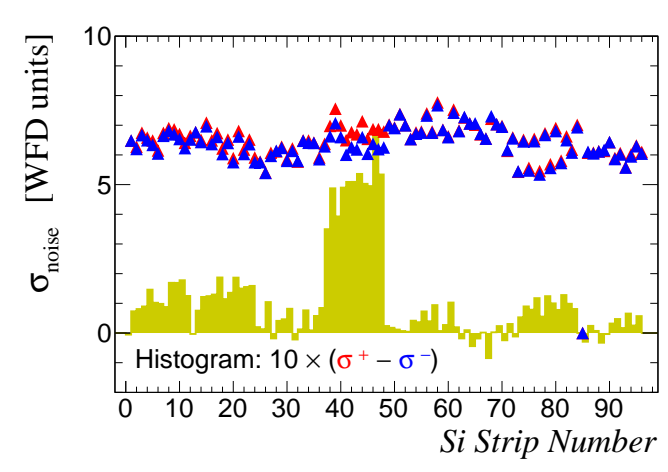

Figure 13: Electronic noise for Jet spin up (red) and down (blue). The difference scaled by factor 10 is shown by the histogram.

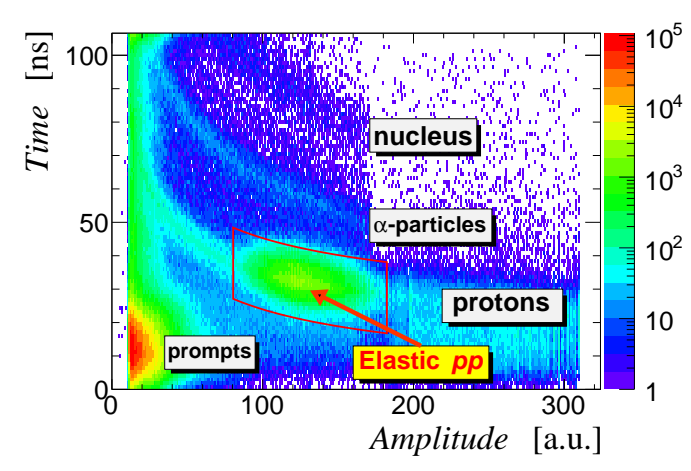

Figure 14: Identification of the acqiured signal and background events. 
2.18). Since the most strong effect is expected in the Blue Right Upper detector, Si strips 36-47, the WFT related systematic error may manifest itself in a measured jet spin asymmetry difference for blue and yellow beams as well as in a discreppancy of asymmetry results with only one Blue Right detecor (Upper or Lower) used in data analysis. In RHIC Run 2017, no evidence of the WFT related systematic error was found. The upper limit, mostly defined by statistical uncertainties in comparing different measurements, was established as

$$
\left(\frac{\sigma_{P}}{P}\right)_{\mathrm{WFT}}^{\mathrm{syst}}<0.2 \%
$$

\subsection{Beam scattering on Oxygen in Jet}

Only about $8 \%$ of all acquired events (see Fig. 14) may be associated with elastic $p p$ scattering. The most intensive component of the background are "prompts" (i.e. the relativistic particles, including beam halo, hitting the Si detectors). The detected prompts, as well as $\alpha$-particles and nucleus (presumably recoil Oxygen from Jet), are kinematically forbidden for the proton beam scattering on proton target in HJET. Thus, we have to conclude that the $\alpha$-particles and nucleous come from the beam $p A$ scattering on Oxygen in Jet (added to dissociator to increase the atomic beam fraction) or, possibly, on Carbon, Nitrogen, and Oxygen in residual beam gas. In case of beam proton scattering on a spectator proton in the $A$ we can observe "recoil-like" protons in HJET detectors. Such protons are expected to expose all Si strip uniformly due to the small solid angle of the HJET detectors and, thus, the related background will be properly subtracted. From the analysis of "'background lines" in Fig. 9 and other similar plots, the corresponding systematic uncertainty were estimated as

$$
\left(\frac{\sigma_{P}}{P}\right)_{\mathrm{pA}}^{\mathrm{syst}}<0.2 \%
$$

\subsection{Molecular hydrogen background}

HJET Breit-Rabi polarimeter actually measures the polarization of atomic protons in Jet. Possible presence of molecular Hydrogen $\left(\mathrm{H}_{2}\right)$ on the beam path effectively dilute Jet polarization $\delta P / P=b_{\mathrm{H}_{2}} /\left(1+b_{\mathrm{H}_{2}}\right)$ where $b_{\mathrm{H}_{2}}$ is atomic mass fraction of the unpolarized $\mathrm{H}_{2}$ to the polarized protons in Jet. There are two main sources of $\mathrm{H}_{2}$ on the beam path:

Molecular Hydrogen in Jet. Out of the HJET dissociator, the ratio of protons in $\mathrm{H}_{2}$ to the polarizad atomic protons is about $10 \%$. Only $0.6 \%$ of these protons entered to the scattering chamber as a part of Jet since the $\mathrm{H}_{2}$ component is not focusing. The fraction of $0.6 \%$ was experimentally evaluated by turning off RF discharge in the dissociator and, thus, by defocusing the atomic Hydrogen. Systematic error in the polarization measurement associated with this unpolarized $\mathrm{H}_{2}$ component may be presented as

$$
\left(\frac{\delta P}{P}\right)_{\text {jet } \mathrm{H}_{2}}^{\text {syst }}=(+0.06 \pm 0.06) \%
$$

Molecular Hydrogen in the scattering chamber. As shown in Fig. 15, molecular hydrogen fills the scattering Chamber 6 by diffusion from the Chamber 7 (scattered and recombined Jet atomic hydrogen) and Chamber 5 (unfocused hydrogen atoms recombined to molecules). We can expect that density profile for molecular hydrogen is much wider than for the atomic hydrogen in Jet. This 


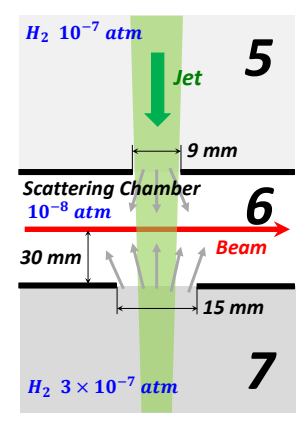

Figure 15: Molecular Figure 16: HJET construction elehydrogen flow in HJET. ment which partialy shadows Si detectors for the beam scattering on $\mathrm{H}_{2}$.

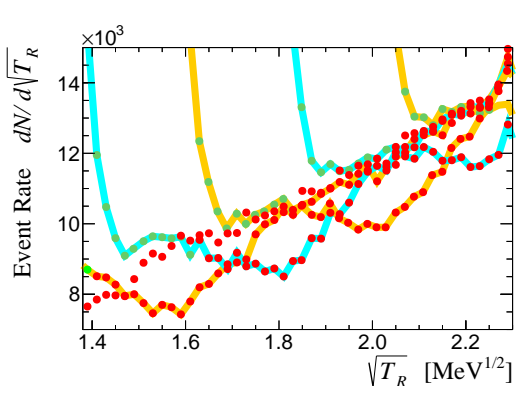

Figure 17: Molecular Hydrogen normalization. $d N / d \sqrt{T_{R}}$ distributions for several consequitive $\mathrm{Si}$ strips.

assumption was verified in a special runs with molecular hydrogen injected to the Chamber 7 and with unpolarized and defocused hydrogen filled Chamber 5.

To evaluate density of $\mathrm{H}_{2}$ we employed a feature of the HJET construction shown in Fig. 16. The recoil protons from the beam scattering on $\mathrm{H}_{2}$ at $z \approx \pm 12 \mathrm{~mm}$ are shadowed by a $d=3 \mathrm{~mm}$ wide wall in the HJET construction. The efficiency of such shadowing is up to $d / d_{\text {str }}=80 \%$ where $d_{\text {str }}=3.7 \mathrm{~mm}$ is the Si strip width. In Fig. 17, the equidistant dips, relative to the strips common background distribution line and seen in every marked Si strip line, are explained by the recoil proton shadowing. Comparing dips with the Jet center elastic event rates (see, for example, Fig. 9) one can evaluate the atomic mass fraction of $\mathrm{H}_{2}$ in Jet center:

$$
b_{\mathrm{H}_{2}}^{(0)}=(0.41 \pm 0.04) \%
$$

It should be noted that the determined forward beam $\mathrm{H}_{2}$ background gives only $15-20 \%$ of the full background rate for the $2-5 \mathrm{MeV}$ recoil protons. Taking into account the backward beam background we have to conclude that the $p A$ contribution to the background rate is $\sim 1.5 \%$ for this energy range (before subtraction).

Taking into account the signal (Gaussian) and the background (flat) distributions within the $\delta \sqrt{T}$ selection cut, the effective (average) $\mathrm{H}_{2}$ background fraction has to be larger and, fot Cuts II, can be estimated as

$$
b_{\mathrm{H}_{2}}=(0.56 \pm 0.06) \%
$$

For almost flat spatial distribution of the $\mathrm{H}_{2}$, the background subtraction, potentially, should work well. However, due to the recoil proton tracking in the Holding Field Magnet, the result is less predictable.

\subsection{Recoil proton tracking in the Holding magnetic field}

Jet polarization is defined by the strength of the holding field magnet located in the scattering chamber. In order to minimize the effect of the holding magnetic field on the recoil protons, The Nested Opposing Helmholtz-type Coils are used (see Fig. 18). The coils currents are adjusted to keep the recoil proton displacement $\delta z=b_{\mathrm{MF}} / \sqrt{T_{R}}$ in the detectors close to zero. The displacement 


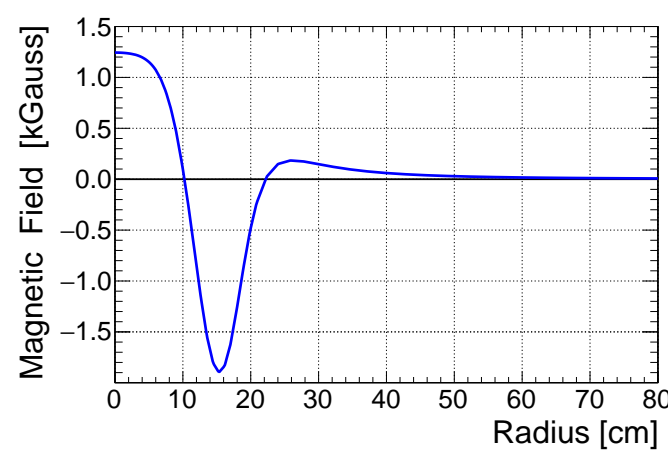

Figure 18: The holding magnetic field as a function of distance from the chamber center on the $x-z$ plane.

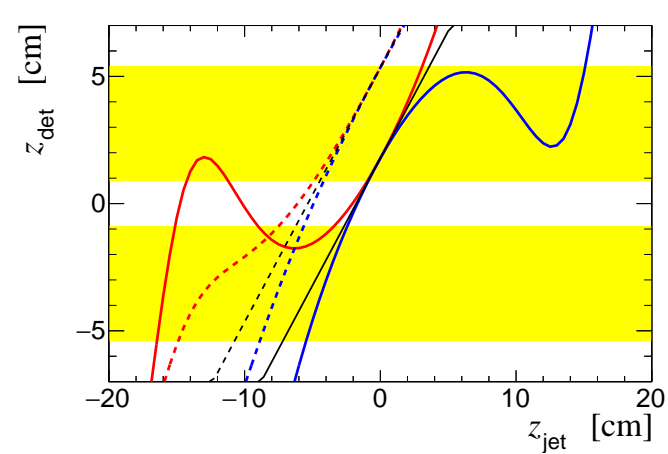

Figure 19: The correlation between $z$-coordinates in the detectors(yellow filled areas) $z_{\text {det }}$ and in a vertex $z_{\text {jet }}$ for $1 \mathrm{MeV}$ (solod lines) and $9 \mathrm{MeV}$ (dashed lines) recoil protons.

is defined by the field integral [9]

$$
\sqrt{2 m_{p}}\left|b_{\mathrm{MF}}\right|=\left(\frac{q L}{c}\right) \times \int_{0}^{L} H(r)\left(1-\frac{r}{L}\right) d r
$$

The adjustment was done assuming the beam scattering in the center of Jet. For the scattering on $\mathrm{H}_{2}$ outside Jet, significant energy dependent displacements broke the basic condition for background subtraction method as shown in Fig. 19. The displacements are different for left-side (blue) and right-side (red). Black straight lines are for the no magnetic field tracking. The simulation of the residual background (after subtraction) is shown in Fig. 20 for forward and backward beams. For a considered detector, forward beam is a beam which polarization is measured by the detector and backward beam is a beam in opposite direction. It has to be pointed out that the residual background is forward/backward beam and left/right detector dependent. Depending on recoil proton energy, the residual background may significantly exceed the level defined by actual $\mathrm{H}_{2}$ density.
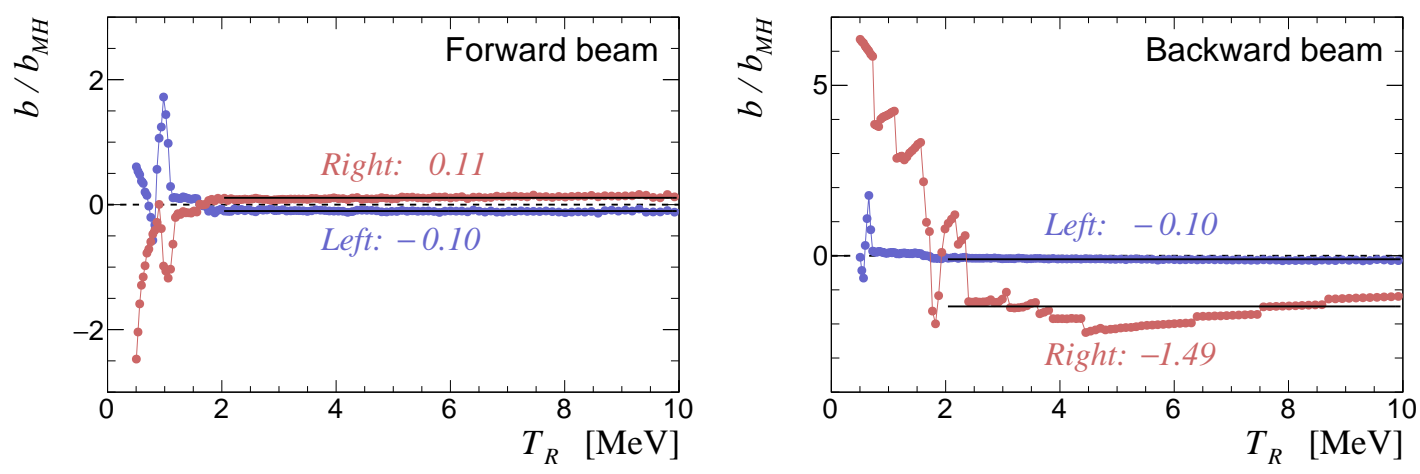

Figure 20: Simulattion of the residual $\mathrm{H}_{2}$ backgrounds for forward and backward beams. Backgrounds in left and right (relative to the forward beam) detectors are shown separately. The average values are given for the $2<T_{R}<10 \mathrm{MeV}$ energy range. 
For the spin pattern used in RHIC, the polarizations of forward and backward beams are uncorrelated in average. This is why the residual $\mathrm{H}_{2}$ background does not affect the measured beam polarization (the ratio $a_{N}^{\text {beam }} / a_{N}^{\text {jet }}$ ).

To evaluate systematic errors in the beam polarization measurement, only residual $\mathrm{H}_{2}$ background produced by forward beam has to be accounted. As one can see in Fig. 20 (left), the residual background is small and almost energy independent for $T_{R}>2 \mathrm{MeV}$. This is why we select the energy cut $T_{R}>2$ in the Cuts II set. For the forward beam left/right residual backgrounds levels shown in Fig. 20 (right), we can evaluate (see Eq. 2.17) that the corresponding systematic corrections to the measured polarization is very small $|\delta P / P| \lesssim 0.01 \%$. Consideration of possible variation of the simulation model does not change this conclusion.

However, there is uncertainty in determining of $\mathrm{H}_{2}$ background caused by the shadowing. This uncertainty may be accounted as the $\mathrm{H}_{2}$ related systematic error:

$$
\left(\frac{\sigma_{P}}{P}\right)_{\text {flat } \mathrm{H}_{2}}^{\text {syst }} \lesssim 0.1 \%
$$

In the data analysis, we corrected the residual $\mathrm{H}_{2}$ background using the simulation for left/right detectors and forward/backward beams.

\subsection{Inelastic $p^{\uparrow} p^{\uparrow} \rightarrow X p$ scattering.}

For inelastic proton beam scattering on a proton target $p p \rightarrow X p$ the recoil (target) proton angle $\theta_{R}$ depends on the kinetic energy as

$$
\tan \theta_{R} \approx \frac{\kappa \sqrt{T_{R}}}{L}\left(1+\frac{m_{p} \Delta}{T_{R} E_{\text {beam }}}\right), \quad \Delta=M_{X}-m_{p} \geq m_{\pi}
$$

The recoil proton energy as a function of the strip position and value of $\Delta$ is shown in Fig. 21. For the $255 \mathrm{GeV}$ beam, only about half $\mathrm{Si}$ strips can be exposed by recoil protons from inelastic scattering. For the detected inelastic events, the beam spin asymmetry is larger than the elastic one while Jet spin asymmetry is smaller (see section 5.4). This is why the inelastic background

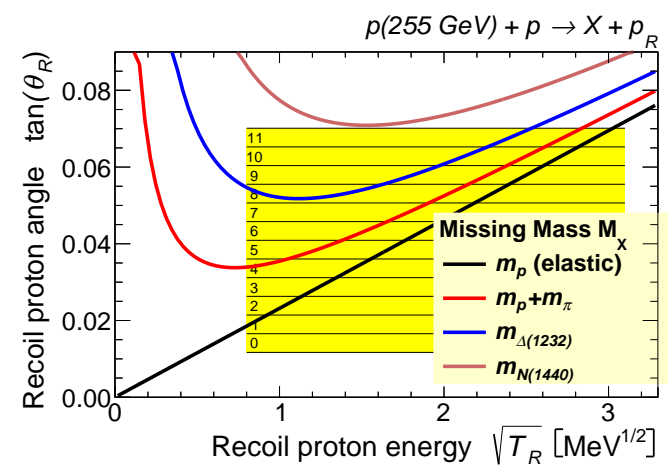

Figure 21: The $p p$ inelastic recoil angle $\theta_{R}$ dependence on kinetic energy $T_{R}$ and missing mass $M_{X}$. The shown lines are effectively smeared with $\sigma_{\theta} \sim 0.0034$ due to Jet size.

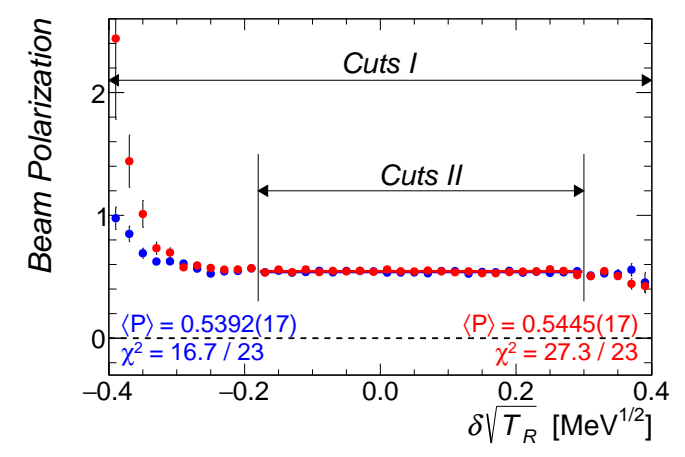

Figure 22: The measured beam polarization dependence on $\delta \sqrt{T}$ event selection cuts. The fit results are given for Cuts II. 
increases the measured beam polarization in HJET. To separate elastic and inelastic $p p$-scattering events we can use the $\delta \sqrt{T}$ cut as shown in Fig. 22. By variation of the cut, it was found that for the optimal cut (included to Cuts II) a possible systematic error may be approximated as

$$
\left(\frac{\delta P}{P}\right)_{p p \rightarrow X p}^{\text {syst }}=(+0.15 \pm 0.15) \%
$$

\subsection{Systematic uncertainties summary}

The systematic uncertainties summary is given in Table 1. For event selection Cuts II, the total correction to compensate a systematic error bias was evaluated as

$$
\tilde{\delta}_{\text {corr }}=(-0.21 \pm 0.37) \%
$$

According to Eq. 4.3, for Jet polarization $\left\langle P_{\text {jet }}\right\rangle=0.957$ the effective Run 2017 beam spin analyzing powers are equal to

$$
\begin{aligned}
\left(A_{N}^{\text {eff }}\right)_{\text {Blue }} & =0.03749 \times\left(1 \pm 0.0034_{\text {stat }} \pm 0.0037_{\text {syst }}\right) \\
\left(A_{N}^{\text {eff }}\right)_{\text {Yellow }} & =0.03739 \times\left(1 \pm 0.0033_{\text {stat }} \pm 0.0037_{\text {syst }}\right)
\end{aligned}
$$

The effective systematic error in the HJET beam polarization measurements was evaluated as

$$
\sigma_{P}^{\text {syst }} / P \lesssim 0.5 \%
$$

For the event selection cuts (3.8), the distributions sensitive to possible systematic uncertainties are shown in Fig. 23. All eight $\chi^{2}$ s are consistent with corresponding numbers of degrees of freedoms. The measured spin dependent asymmetry slopes are consistent with each other. The average value is $\beta_{5}=(7.9 \pm 0.6) \times 10^{-3}\left(\chi^{2} / \mathrm{NDF}=4.2 / 3\right)$. Thus, these distributions do not indicate unaccounted systematic errors.

Following the criteria formulated in section 2.4, the recoil energy range may be extended to $0.7 \mathrm{MeV}$ for $a_{N}^{\text {beam }}, \lambda_{\text {beam }}$, and $\lambda_{\text {jet }}$ distributions. A clearly seen low energy $\left(T_{R}<22 \mathrm{MeV}\right.$ discrepancy for the $a_{N}^{\text {jet }}$ looks like an overestimate of the forward beams $\mathrm{H}_{2}$ background. However,

\begin{tabular}{l|c|c} 
Source & $\delta P / P[\%]$ & $\sigma_{P} / P[\%]$ \\
\hline \hline Long term stability & & 0.1 \\
Jet Polarization & & 0.1 \\
Jet $\mathrm{H}_{2}$ & +0.06 & 0.06 \\
Flat $\mathrm{H}_{2}$ & & $\lesssim 0.1$ \\
$p \mathrm{~A}$ & & $\lesssim 0.2$ \\
$p p \rightarrow X p$ & +0.15 & 0.15 \\
WFT & & $\lesssim 0.2$ \\
\hline Total & +0.21 & $\lesssim 0.37$
\end{tabular}

Table 1: Systematic uncertainties summary for the event selection Cuts II. Some systematics uncertainties, such as corrections due to the vertical size of the detectors, possible uncertainty in the energy calibration are canceled in the beam/Jet asymmetry ratio and, therefore were not included to the summary. 

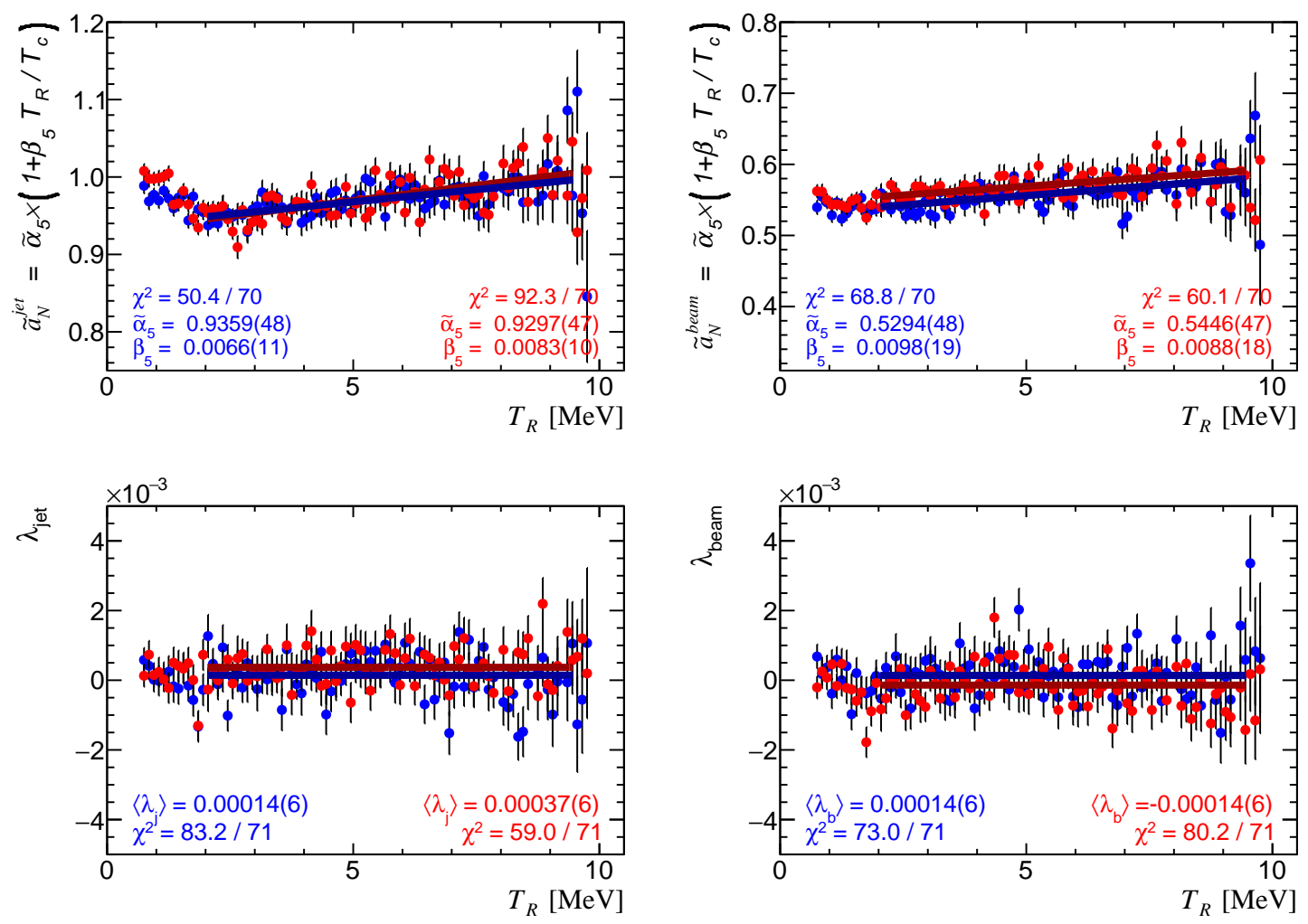

Figure 23: The spin and integrated intensity asymmetries as functions of $T_{R}$ for event selection Cuts II. The fit results are given for the energy range $2 .<T_{R}<9.5 \mathrm{MeV}$.

it should be noted that, at the moment, we have no satisfactory model to describe the phenomena. A similar, but several times smaller effect, was also observed for $100 \mathrm{GeV}$ proton beams in RHIC Run 2015.

\section{HJET performance in RHIC Run 2017}

RHIC operation in 2017 included 14 weeks of $255 \mathrm{GeV} p^{\uparrow} p^{\uparrow}$ run and 3 weeaks of $27.2 \mathrm{GeV} / n$ Gold-Gold run.

\subsection{Monitoring of the proton beams polarization}

A typical result of the beam polarization measurement for an 8-hour store may be presented as

$$
\left\langle P_{\text {beam }}\right\rangle=\left(\sim 56 \pm 2.0_{\text {stat }} \pm 0.3_{\text {syst }}\right)
$$

The store average polarizations for every RHIC fill are displayed in Fig. 24. Running conditions were changed several times during the Run to optimize RHIC performance. The dependence of the average beam polarization on the running conditions are summarized in Table 2. 


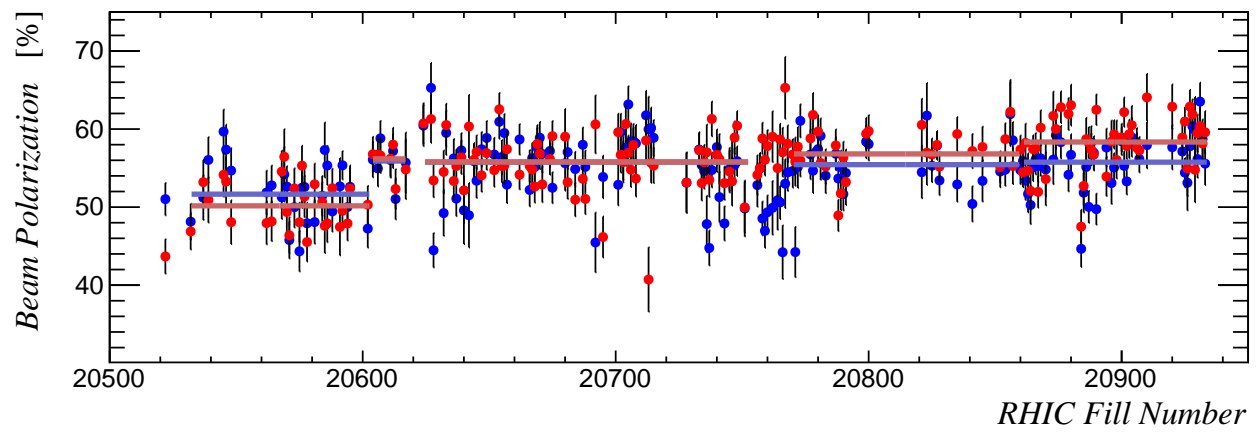

Figure 24: Monitoring of the RHIC store average absolute polarization of the proton beams in Run 2017. Only statistical errors are shown.

\begin{tabular}{l|c|c|l} 
RHIC Fills & Blue Beam & Yellow Beam & Running Conditions[10, 11] \\
\hline $20537-20603$ & $51.7 \pm 0.5$ & $50.2 \pm 0.5$ & AGS Single Harmonic \\
$20604-20617$ & $55.9 \pm 0.9$ & $56.2 \pm 0.9$ & AGS Dual Harmonic \\
$20624-20753$ & $55.8 \pm 0.3$ & $55.8 \pm 0.3$ & slower Ramp \\
$20770-20860$ & $55.6 \pm 0.3$ & $56.8 \pm 0.4$ & D' Lattice only in Blue beam \\
$20861-20935$ & $55.4 \pm 0.4$ & $58.3 \pm 0.3$ & \\
\hline Run 2017 average & 54.9 & 56.1 &
\end{tabular}

Table 2: The average beams polarizations depending on the running conditions. The shown errors are statistical uncertainties in determination of the interval average polarization.

\subsection{Single spin-flip analyzing power in elastic $\boldsymbol{p}^{\uparrow} \boldsymbol{p}^{\uparrow}$ scattering}

For the $p^{\uparrow} p$ single spin-flip analyzing power measurement we used the Run average Jet polarization

$$
P_{\text {jet }}=0.954 \pm 0.003_{\text {syst }}
$$

This value includes a correction due to the vertical size of the detectors $\left\langle\left|\sin \varphi_{L, R}\right|\right\rangle \approx 0.997$. The error in Eq. 5.2 accumulates all entries from Table 1 except for inelastic $p p \rightarrow X p$ contribution. Since inelastic background correction has different signs for Jet $a_{N}^{\text {jet }}$ and beam $a_{N}^{\text {beam }}$ spin asymmetries, the related systematic uncertainty in $A_{N}(t)$ measurements should be evaluated directly in the analyzing power fit.

To measure analyzing power (2.12) we determined $\operatorname{Re} r_{5}$ and $\operatorname{Im} r_{5}$ from the data fit

$$
a_{N}\left(T_{R}\right)=A_{N}\left(t, \operatorname{Re} r_{5}, \operatorname{Im} r_{5}\right)
$$

Jet spin asymmetry $a_{N}^{\text {jet }}\left(T_{R}\right)$ was measured in the energy range $1.9<T_{R}<9.9 \mathrm{MeV}$. Jet polarization (5.2) was a fixed parameter in the fit. The beam spin asymmetry $a_{N}^{\text {jet }}\left(T_{R}\right)$ range was $0.7<T_{R}<$ 9.9 MeV. The average blue and yellow beams polarizations were considered as free parameters in the fit. The preliminary results are shown in Fig. 25.

Several systematic errors which were effectively canceled in the beam polarization measurement has to be considered 

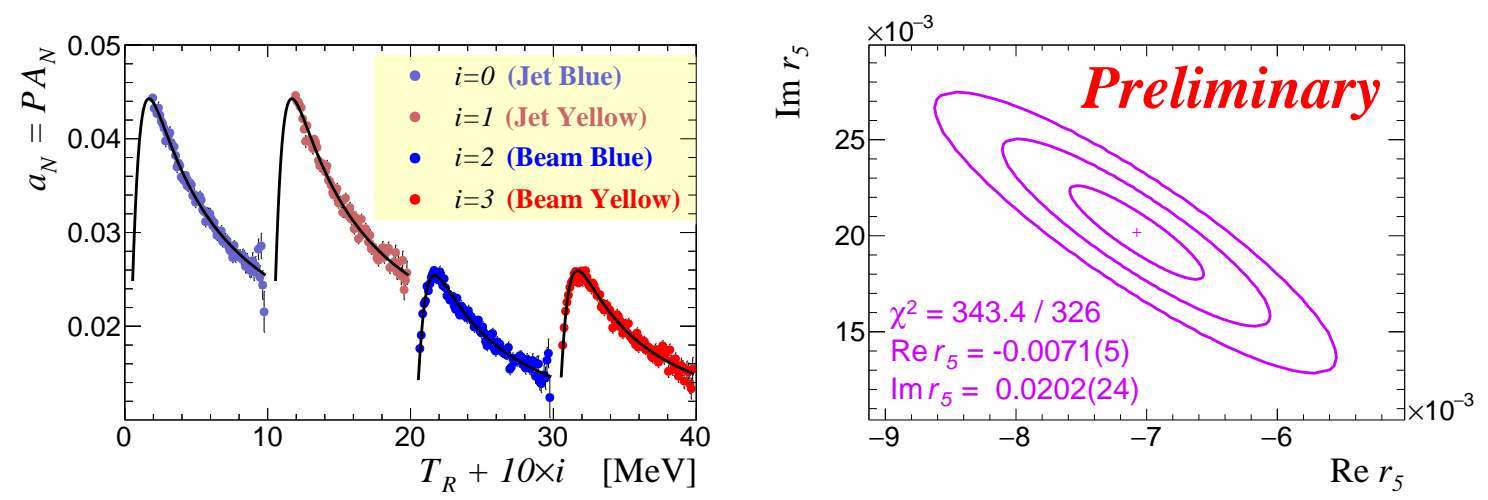

Figure 25: Left: The measured Jet and the beam spin asymmetries used in the $A_{N}(t)$ fit. Right: Fitted value of $r_{5}$ with the $1-\sigma, 2-\sigma$, and $3-\sigma$ statistical error contours.

(i) The systematic uncertainties due to inefficiency of $\mathrm{H}_{2}$ background subtraction (including backward beam background).

(ii) Possible systematic uncertainties in energy calibration.

(iii) The analyzing power fit is very sensitive to the real to imaginary amplitude ratio $\rho$ which is considered as an external parameter known from inelastic $p p$ scattering experiments.

According to the preliminary consideration, the systematic errors in determination of $\operatorname{Re} r_{5}$ and $\operatorname{Im} r_{5}$ are comparable with the statistical ones.

\subsection{Double spin-flip analyzing power in elastic $\boldsymbol{p}^{\uparrow} \boldsymbol{p}^{\uparrow}$ scattering}

Double spin-flip analyzing power may be expressed via measured asymmetries as

$$
A_{N N}\left(T_{R}, r_{2}\right)=\frac{A_{N}^{2}\left(T_{R}, r_{5}\right)}{a_{N}^{\text {beam }}\left(T_{R}\right)} \frac{a_{N N}\left(T_{R}\right)}{a_{N}^{\text {jet }}\left(T_{R}\right)}
$$
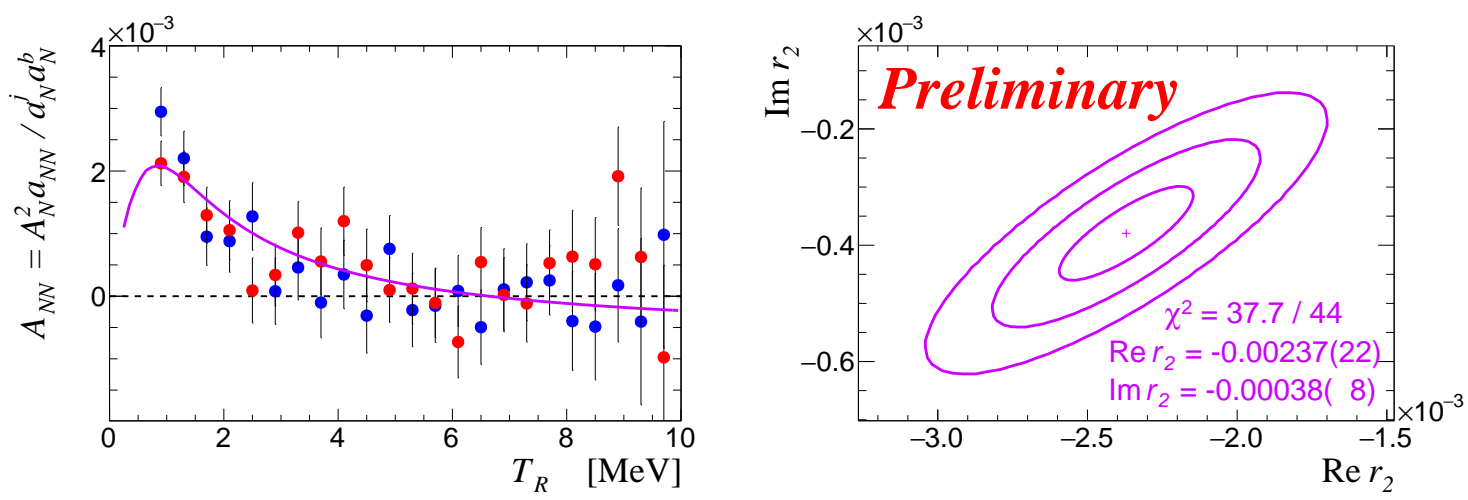

Figure 26: Left: The measured double spin-flip analyzing power for blue and yellow beams and the combined fit. Right: Fitted value of $r_{2}$ with the $1-\sigma, 2-\sigma$, and 3- $\sigma$ confidence contours. 
The results of the fit against $\operatorname{Re} r_{2}$ and $\operatorname{Im} r_{2}$ are shown in Figs. 26. It has to be pointed out that Jet spin related systematic errors are canceled in the ratio $a_{N N} / a_{N}^{\text {jet }}$. This cancellation was verified by comparing the ratio measured with and without background subtraction. For the beam spin asymmetry, $a_{N}^{\text {beam }}$, the systematic uncertainties are small in the full energy range $0.7<T_{R}<$ 9.9 MeV. The used single spin-flip $A_{N}(t)$ was determined with high precision (see above) forthe same HJET data. Since $\left|a_{N N}\right| \ll a_{N}^{\mathrm{b}, \mathrm{j}}$, we can conclude that error in measurement of $r_{2}$ is strongly dominated by statistical fluctuations.

\subsection{Evaluation of analyzing power in inelastic $p^{\uparrow} p^{\uparrow} \rightarrow X p$ scattering.}

In case of $255 \mathrm{GeV}$ proton beam, as it was discussed above, HJET is also hit by the recoil protons from inelastic $p^{\uparrow} p^{\uparrow} \rightarrow X p$ scattering. In spite of low statistic of a few percent relative to the elastic events, the inelastic events can be well isolated. We can employ this data to study spin correlated asymmetries in the inelastic scattering for $4 \lesssim-t \lesssim 10 \mathrm{GeV}^{2}$ and $M_{X} \lesssim 1.4 \mathrm{GeV}$. The results of a very preliminary study are shown in Fig. 27. The diagonals in the pictures are populated mostly by high intensity elastic events. In a glance one can see that

(i) Inelastic statistics are dominated by $p p \rightarrow N(1420) p$ scattering.

(ii) Jet spin analyzing power is much smaller (factor 10-15) compared to the beam spin analyzing power.

(iii) The analyzing power has a maximum around $t=-0.004 \mathrm{GeV}^{2}$ and $M_{X} \sim M_{\Delta(1232)}$ both for the beam and jet asymmetry.

(iv) For the beam spin asymmetry, the analyzing power is large $A_{N}^{\text {beam }}>15 \%$ at the maximum. Additional study is still needed to be more conclusive.

\subsection{Analyzing power in $p^{\uparrow} \mathrm{Au}$ scattering}

Scattering of a nuclei with energy $E_{\text {beam }}$ (per nucleon) on a transversely polarized proton target (Jet) is equivalent to scattering of the polarized proton with energy

$$
E_{p}=E_{\text {beam }} \frac{m_{p} N}{M} \approx E_{\text {beam }}
$$

on the nuclei target. $M$ is the nuclei mass and $N$ is number of nucleons in the it. The recoil proton angle dependence on kinetic energy is very similar to the elastic $p p$ one (3.4) with only a minor
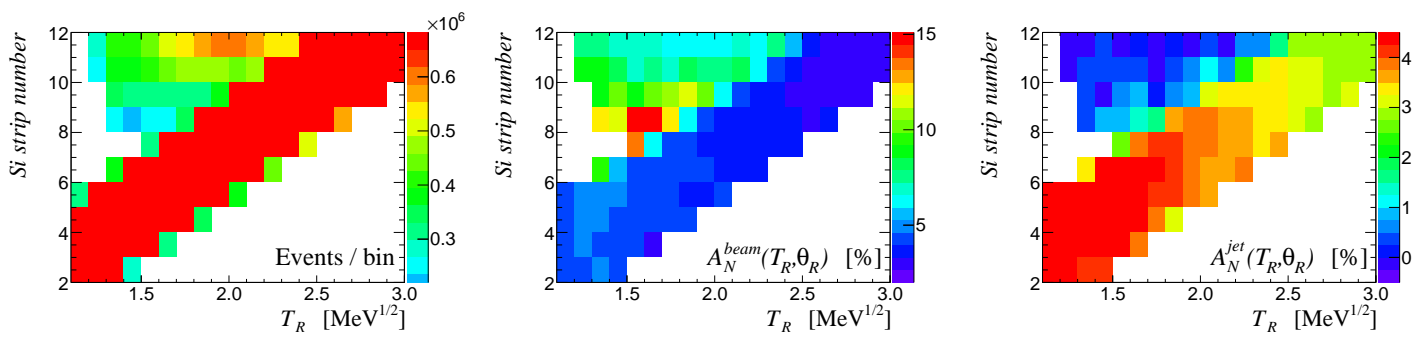

Figure 27: Study of the spin dependent asymmetries in inelastic $p^{\uparrow} p^{\uparrow} \rightarrow X p$ scattering. The diagonals are strongly dominated by elastic events. The inelastic events are above the diagonal. Left: Statistics per bin (background subtracted). Jet center statistics for elastic events is (1.5-2) $\times 10^{7}$ events/bin. Center: Measured beam spin asymmetry. Right: Measured Jet spin asymmetry. 


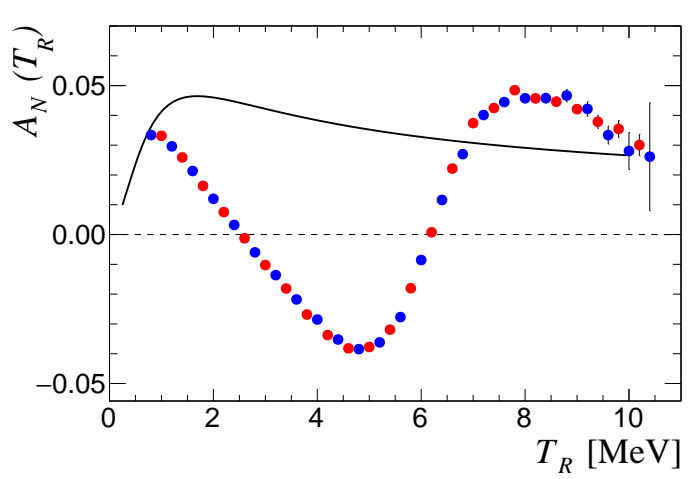

Figure 28: Measured analyzing power for $p^{\uparrow} \mathrm{Au}$ $\left(E_{\mathrm{Lab}}=27.2 \mathrm{GeV}\right)$ separately for blue and yellow beams. $\quad p^{\uparrow} p$ analyzing power (solid black line) is shown to define the scale.

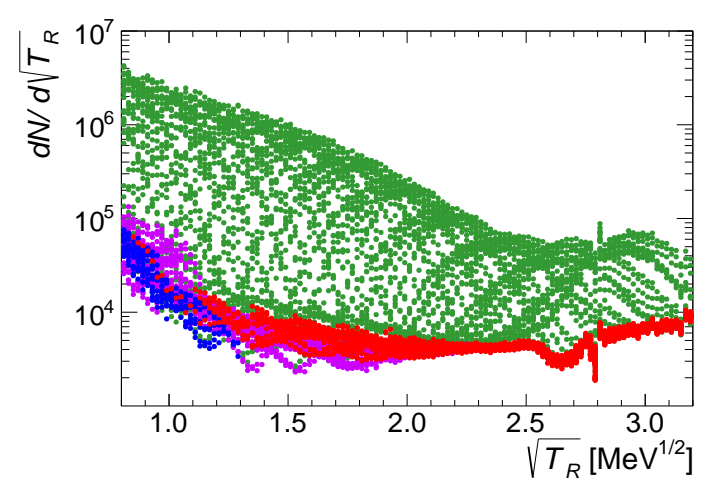

Figure 29: Search for the inelastic $\mathrm{Au} p \rightarrow X p$ events contribution. Color indexes are the same as in Fig. 9.

correction

$$
\tan \theta_{R}=\sqrt{\frac{T}{2 m_{p}}} \sqrt{\frac{E_{p}+\mu}{E_{p}-\mu\left(1+\frac{T}{m_{p}}\right)-\frac{m_{p}^{2}-\mu^{2}}{E_{p}-\mu}\left(1+\frac{T}{2 m_{p}}\right)}} \approx \sqrt{\frac{T}{2 m_{p}}}
$$

where $\mu=m_{p}^{2} / M$. Therefore, HJET can actually be used in a standard way to measure analyzing power of polarized proton scattering on the nuclei target for any ions stored in the RHIC. For the Run $201727.2 \mathrm{GeV} / n$ Gold beams, the measured $A_{N}(t)$ is shown in Fig. 28.

To evaluate possible inelastic scattering $\mathrm{Au} p \rightarrow X p$, we plotted the superposition of the $d N / d \sqrt{T_{R}}$ as it was done for the $p p$ scattering in Fig. 9. In a glance (see Fig. 9), a possible contamination of the elastic data by the inelastic Au $p \rightarrow X p$ events does not exceed $\lesssim 1 \%$ level.

\section{Summary}

The HJET upgrade in Run 2015 and the developed methods of the data analysis allowed us to significantly improve the polarimeter performance. The fluctuation of systematic errors during 3 months of RHIC Run 2017 did not exceed $0.1 \%$. The systematic uncertainty in the measurement of the absolute, RHIC store average polarization was evaluated to be $\lesssim 0.5 \%$ for both RHIC beams. By the product, HJET allowed us to make high precision study of the single spin-flip and double spin-flip analyzing powers in elastic $p^{\uparrow} p^{\uparrow}$ scattering at $\sqrt{s}=21.1 \mathrm{GeV}$. Also, it has been shown that spin correlated asymmetries in inelastic $p^{\uparrow} p^{\uparrow} \rightarrow X p$ scattering, as well as in $p^{\uparrow} \mathrm{Au}$ scattering can be studied.

\section{References}

[1] E. Aschenauer et al., arXiv:1501.01220.

[2] A. Zelenski et al., Nucl. Instrum. Meth. A 536, 248 (2005). 
[3] A. Poblaguev, PoS PSTP 2015, 032 (2015).

[4] J. Ashkin et al., AIP Conf. Proc. 42, 142 (1978). doi:10.1063/1.31281

[5] N.H. Buttimore et al., Phys. Rev. D 59, 114010 (1999); N.H. Buttimore et al., Phys. Rev. D 64, 094021 (2001).

[6] D. A. Fagundes, M. J. Menon and P. V. R. G. Silva, Nucl. Phys. A 966, 185 (2017); C. Patrignani et al (Particle Data Group), Chin. Phys. C 40 (2016) 100001.

[7] H. Dong et al., 2007 IEEE Nuclear Science Symposium Conference Record, Honolulu, HI, 2007, pp. 831-833. doi:10.1109/NSSMIC.2007.4436457.

[8] A. Poblaguev, BNL-104-366-2014-IR (February 2014),

[9] A. Poblaguev, BNL-104-363-2014-IR (March 2014),

[10] H. Huang et al., in Proc. 8th Int. Particle Accelerator Conf. (IPAC'17), Copenhagen, Denmark, May 2017, paper TUPVA149, pp. 2452-2454, http://jacow.org/ipac2017/papers/tupva149.pdf.

[11] V.H. Ranjbar et al., in Proc. 8th Int. Particle Accelerator Conf. (IPAC'17), Copenhagen, Denmark, May 2017, paper TUPVA050, pp. 2188-2190, http://jacow.org/ipac2017/papers/tupva050.pdf. 